\title{
Comparison of prasugrel and clopidogrel in the setting of ACS without revascularization
}

In patients with acute coronary syndrome (ACS) who have not undergone revascularization, but are receiving aspirin, prasugrel is associated with a similar rate of major cardiovascular events and has a similar safety profile as clopidogrel when used over the long term ( 15 months). These 'neutral' findings from the TRILOGY ACS trial were presented at the 2012 European Society of Cardiology Congress and published simultaneously in the New England Journal of Medicine. Because clopidogrel is off patent and, therefore, is currently much cheaper than prasugrel, many clinicians believe that clopidogrel will remain the preferred antiplatelet agent in this patient population.

A substantial proportion of patients with ACS are treated medically without revascularization, but this patient population has been under-represented in contemporary, large, randomized, controlled trials. "Patients who are medically managed are at higher risk for repeated cardiovascular-related events," explains Professor Magnus Ohman (Duke University Medical Center, Durham, NC, USA), "so optimizing medical therapy for these patients is extremely important." The TRILOGY ACS trial, for which Ohman is study chairman, was therefore designed to compare aspirin plus prasugrel with aspirin plus clopidogrel in patients with unstable angina or myocardial infarction without ST-segment elevation.

The primary analysis in the randomized, double-blind, double-dummy, event-driven trial was to compare $10 \mathrm{mg}$ prasugrel ( $5 \mathrm{mg}$ in patients weighing $<60 \mathrm{~kg}$ ) daily with $75 \mathrm{mg}$ clopidogrel daily in patients aged $<75$ years. A secondary analysis involving those in the primary analysis as well as individuals aged $\geq 75$ years, who received $5 \mathrm{mg}$ prasugrel or $75 \mathrm{mg}$ clopidogrel daily, was also undertaken. In total, 7,243 patients aged $<75$ years and 2,083 patients aged $\geq 75$ years were enrolled into the trial at 966 sites in 52 countries.
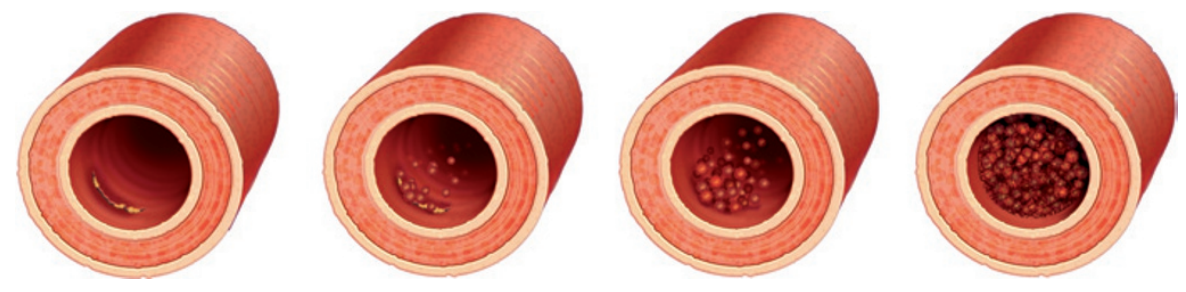

() Mart2216 | Dreamstime.com

Trial participants remained on the study drug for median 14.8 months. During follow-up of up to 30 months (median 17.1 months), $24 \%$ of the prasugrel group and $22 \%$ of the clopidogrel group $(P=0.03)$ discontinued their assigned treatment, and $7.9 \%$ of the patients aged $<75$ years underwent revascularization.

Among the patients aged $<75$ years, $13.9 \%$ of the prasugrel group and $16.0 \%$ of the clopidogrel group (HR 0.91, 95\% CI 0.79-1.05, $P=0.21$ ) experienced death from cardiovascular causes, nonfatal myocardial infarction, or nonfatal strokethe composite primary end point-during follow-up. Among the entire study population, $18.7 \%$ of individuals assigned to prasugrel and $20.3 \%$ of patients assigned to clopidogrel (HR 0.96, 95\% CI 0.86-1.07, $P=0.45)$ experienced such an event.

Interestingly and unexpectedly, KaplanMeier curves for the primary end point overlapped for the first 12 months of treatment in the patients aged $<75$ years, but then seemed to diverge somewhat. In timedependent analysis, the TRILOGY ACS investigators found a weak, nonsignificant trend toward reduced risk with prasugrel after 12 months (HR for $\leq 12$ months of treatment $0.99,95 \%$ CI $0.84-1.16$; HR for $>12$ months of treatment $0.72,95 \%$ CI $0.54-$ 0.97; $P=0.07$ for interaction). Moreover, in the patients aged $<75$ years, prespecified analysis indicated that prasugrel might be associated with a reduced risk of multiple recurrent ischemic events compared with clopidogrel (HR 0.85, 95\% CI 0.72-1.00, $P=0.04)$. Time-dependent analysis showed that prasugrel was associated with reduced risk of recurrent ischemic events after 12 months (HR for $<12$ months of treatment $0.94,95 \%$ CI $0.79-1.12$; HR for $\geq 12$ months of treatment $0.64,95 \%$ CI $0.48-0.86$; $P=0.02$ for interaction). The investigators believe that these time-dependent findings deserve further exploration.

In the patients aged $<75$ years, prasugrel and clopidogrel were associated with similar rates of non-CABG-related GUSTO severe and life-threatening bleeding ( $0.9 \%$ vs $0.6 \%, P=0.87)$ and TIMI major bleeding ( $2.1 \%$ vs $1.5 \%, P=0.27)$. The same was true in the entire trial population $(1.1 \%$ vs $1.0 \%, P=0.53$, and $2.5 \%$ vs $1.8 \%, P=0.29$, respectively). The only noted statistically significant differences in adverse events in individuals aged $<75$ years were a higher risk of non-CABG-related TIMI major or minor bleeding with prasugrel $(3.3 \%$ vs $2.1 \%, P=0.02)$ and a higher risk of heart failure with clopidogrel (1.3\% vs $1.8 \%$, $P=0.045)$. In the entire trial population, only the rates of anemia (2.6\% for prasugrel vs $1.8 \%$ for clopidogrel, $P=0.013$ ) and peripheral edema ( $1.9 \%$ for prasugrel vs $2.7 \%$ for clopidogrel, $P=0.012$ ) differed between the two treatment groups.

The TRILOGY ACS investigators conclude their trial report by reminding us that "the optimal treatment duration and intensity of $\mathrm{P} 2 \mathrm{Y}_{12}$ inhibition after a coronary event for patients who do not undergo revascularization remain uncertain".

Bryony M. Mearns

Original article Roe, M. T. et al. Prasugrel versus clopidogrel for acute coronary syndromes without revascularization. N. Engl. J. Med. doi:10.1056/NEJMoa1205512 